\title{
An updated quantitative evaluation of the association between leukaemia risk and XRCC3 Thr241Met polymorphism
}

\author{
Emmanuel Kwateng Drokow ${ }^{1}$, Clement Yaw Effah², Kate Benedicta Amenador ${ }^{3}$, Clement Agboyibor ${ }^{3}$, \\ Gloria Selorm Akpabla ${ }^{4}$, Hafiz Abdul Waqas Ahmed ${ }^{5}$, Juanjuan Song ${ }^{5}$, Kai Sun ${ }^{5,6}$
}

\author{
${ }^{1}$ Department of Radiation Oncology, Zhengzhou University People's Hospital \& Henan \\ Provincial People's Hospital Henan, China \\ ${ }^{2}$ College of Public Health, Zhengzhou University, Zhengzhou, China \\ ${ }^{3}$ Department of Applied Linguistic and Foreign Languages, Zhengzhou University, \\ Zhengzhou, China \\ ${ }^{4}$ School of Pharmaceutical Sciences, Zhengzhou University, Zhengzhou, China \\ ${ }^{5}$ Department of Internal Medicine, Tianjin Medical University, Tianjin, China \\ ${ }^{6}$ Department of Haematology, Zhengzhou University People's Hospital \& Henan \\ Provincial People's Hospital Henan, China"
}

Submitted: 27 February 2021

Accepted: 26 March 2021

Arch Med Sci

DOI: https://doi.org/10.5114/aoms/135003

Copyright (c) 2021 Termedia \& Banach

\begin{abstract}
Introduction: Several analyses have been conducted to assess the association between leukaemia risk and XRCC3 Thr241Met polymorphism. However, their results are conflicting. Hence, this comprehensive study was carried out to obtain a more accurate assessment of the association between leukaemia risk and XRCC3 Thr241Met (rs861539) polymorphism

Material and methods: We searched Ovid, Chinese Biomedical Literature Database (CBM), China National Knowledge Infrastructure (CNKI), Google Scholar, PubMed database and Excerpta Medica Database (EMBASE) for potential eligible studies published as of July 2020. Pooled odds ratio (OR) and $95 \%$ confidence interval $(\mathrm{Cl})$ were used in evaluating the strength of the association between Thr241Met polymorphism of XRCC3 and leukaemia, and the $p$-value less than $0.05(p<0.05)$ was considered as the level of significance.

Results: A total of 17 studies consisting of 7241 controls and 4198 cases met our inclusion criteria. No significant association was observed between leukaemia risk and Thr241Met polymorphism of XRCC3 across the five genetic variants. In our ethnicity subgroup evaluation, we noted a significant association between leukaemia risk and Thr241Met polymorphism of XRCC3 among Caucasians under four genetic models. Furthermore, SNP (rs861539) of XRCC3 has a protective effect among Asians under four genetic models. Conclusions: Thr241Met polymorphism of XRCC3 has a protective effect in the Asian population but has oncogenic potential in the Caucasian population.
\end{abstract}

Key words: XRCC3, Thr241Met, leukaemia, polymorphism, meta-analysis.

\section{Introduction}

Leukaemia is described as a group of progressive cancerous disorders of the haematopoietic system with an elevated or abnormal number of immature leukocytes or white blood cells (WBC) formed by the bone marrow. This

\author{
Corresponding author: \\ Kai Sun \\ Department of Haematology \\ Zhengzhou University \\ People's Hospital \& \\ Henan Provincial \\ People's Hospital \\ Henan, 7 Weiwu Road \\ Jinshui District \\ 450001 Zhengzhou \\ Henan, China \\ Phone: +86-18237110038 \\ E-mail: sunkai@cellscience.org
}


inhibits natural development of blood cells, causing anaemia thrombocytopenia, as well as other clinical disorders [1-4]. The prevalence and mortality rate of leukaemia in 2020 were estimated at 60,530 and 35,470 respectively; accounting for $3.4 \%$ of all new cancer cases and $3.8 \%$ of all cancer death worldwide [5]. The global incidence rate of leukaemia continues to rise due to its complex and vast characteristics contributing to its poor prognosis [6]. The four key forms of cytogenetic-based leukaemia are acute myeloid leukaemia, acute lymphocytic leukaemia, chronic lymphocytic leukaemia, and chronic myeloid leukaemia [7-10]. The occurrence of leukaemia has been linked with many risk factors, including genetic variations, chromosome $21 \mathrm{q}$ abnormalities, Fanconi anaemia, cytotoxic drug usage and ionising radiation exposure [11-13].

DNA repair mechanisms plays an essential role in sustaining the stability and integrity of genomes. It is generally established that abnormalities in repair mechanisms are linked to genomic instability, which can progressively stimulate the growth of certain cancers including leukaemia [14]. X-ray repair cross-complementing group 3 (XRCC 3 ) is composed of some polymorphic genes which belongs to one of the key proteins in the DNA repair pathways, which is important for stability of the genome by stimulating the search for homology and spurring the single-stranded tails invasion into the homologous chromatid DNA double helix [15]. XRCC3 is positioned at chromosome 14 (human) band 14q32.33 (chromosome 14q32.33), is actively involved in the repair of DNA, and participates in homologous recombination (HR) of DNA double-strand breaks and cross-links [16, 17]. During the repair of the homologous recombination, the protein XRCC3 directly interacts with and stabilises the protein RAD51 [18-21]. A variety of genes which are engaged in repairing and preserving double-strand breaks have been found with genetic polymorphisms which involve genes that belong to the homologous recombination DNA double-strand breaks repair pathways [22].

The polymorphism in the XRCC3 gene at codon 241 leads to the substitution of amino acid threonine (Thr)-to-methionine (Met), which is an unconventional improvement [23]. The substitution of amino acid Thr241Met attributable to the exon 7 transition of $18607 \mathrm{C} / \mathrm{T}$ in the XRCC3 gene was observed to function effectively, since it appears to be related to a number of elevated micronuclei in the peripheral blood lymphocytes of humans exposed to radiation $[24,25]$ and is also reported to be related with malignancies [26]. The operational importance of this shift in amino acids has not been clearly determined, even though some studies have noted significant relationships between cancers (such as oesophageal cancer, bladder cancer, breast cancer, colorectal) and this variant genotype [27-30]. This researched focused on $18067 \mathrm{C} / \mathrm{T}$ (rs861539), which is a single nucleotide polymorphism (SNP) in the X-ray repair cross-complementing group 3 gene [31]. The XRCC3 Thr241Met polymorphisms arises in three genetic variants: and homozygous (TT), heterozygous (CT), and wild-type (CC) genotypes.

Several analyses have been conducted to assess the relationship between leukaemia risk and XRCC3 Thr241Met polymorphism. However, their results are conflicting [31-33]. A study by Qin et al. appears not to be void of selection bias because only three databases (CNKI, EMBASE and PubMed) were used by the authors in their search for the relevant literature [31]. Furthermore, no flow chart showing the literature selection process was presented. Hence, this comprehensive study was carried out to obtain a more accurate assessment of the association between leukaemia risk and XRCC3 Thr241Met (rs861539) polymorphism.

\section{Material and methods}

\section{Identification and eligibility of relevant studies}

We searched Ovid, Chinese Biomedical Literature Database (CBM), China National Knowledge Infrastructure (CNKI), Google Scholar, PubMed database and Excerpta Medica Database (EMBASE) and grey literature for the potential eligible studies that had been published as of July 2020. The search utilized the following keywords: "leucocythemia" or "leukocythemia", "X-ray repair cross-complementing group 3" or "XRCC3", "leukaemia" and "variant or polymorphism". Additionally, we included other possible publications identified via manual search. This methodology is described in our previous studies [34, 35].

\section{Inclusion and exclusion eligibility}

The inclusion criteria of our studies were as follows: 1) cohort or case-control studies, 2) studies assessing the relationship between the risk of leukaemia and Thr241 polymorphism of XRCC3, 3) articles with the distribution of the control genotype in Hardy-Weinberg equilibrium, and 4) studies with adequate information for estimating odds ratios (ORs) and $95 \%$ confidence intervals $(\mathrm{Cls})$. The exclusion criteria were as follows: 1) studies with incomplete data, 2) non-casecontrol studies, and 3) duplication of past publication.

\section{Data extraction}

Two reviewers independently extracted the data by following the standardised protocol and procedure for data extraction. Another investigator also participated in the extraction of data. Any disagreements and discrepancies were resolved through full discussion with the third reviewer. The extracted information comprised: the year of publication, the name of the first author, cases and controls genotype 
distribution, ethnicity, country of origin, the control source, sample sizes, and methods for genotyping.

\section{Quality score evaluation}

The Newcastle-Ottawa Scale (NOS) for evaluating the quality of non-randomized studies was used in our meta-analyses. Two reviewers independently evaluated the quality of studies in accordance with the quality assessment scale. The cancer genetic factors and epidemiological factors were the basis for these scores. Any dispute between the reviewers was settled through dialogue. We used the aggregate score of quality items to determine the overall quality level in primary research. The range for the total score was from zero (low) to fifteen (high). A total score less than 5 was considered to be poor quality and was further excluded.

\section{Statistical analysis}

Pooled odds ratio (OR) and 95\% confidence interval $(\mathrm{Cl})$ were used in evaluating the strength of the association between Thr241Met polymorphism of XRCC3 and leukaemia risk. The genotypes and alleles were compared using five different genetic models: allele contrast model ( $T$ vs $C$ ), dominant model ( $T T+T C$ vs $C C$ ), recessive model ( $T T$ vs $T C+C C$ ), over-dominant (TC vs TT+CC), as well as homozygote (TT vs CC) models. Hardy-Weinberg equilibrium was estimated in the various control groups for each study using the $\chi^{2}$ test, and the $p$-value less than $0.05(p<0.05)$ was considered as the level of significance. The heterogeneity between the studies was evaluated by the index $I^{2}$. A high value of $I^{2}(>50 \%)$ indicated heterogeneity. If heterogeneity was not present $\left(I^{2}<50 \%\right)$, a fixed-effect model was applied for analysis; otherwise, a random effect model was adopted. The outcomes of the overall odds ratio for all the included studies under the five genetic models were presented using forest plots. We conducted subgroup analyses based on ethnicities, control source and method of genotyping. Analysis of sensitivity was carried out to ascertain the impact of individual data sets on the combined estimates. Egger's test and Begg's funnel plot were used to assess bias in the publication. The Web tool MetaGenyo, Version 12.0. was used in all our analyses. MetaGenyo provides a detailed and complete workflow, which can be implemented without programming skills in an easier-to-use setting. In addition, MetaGenyo was designed to guide users via the key stages of a genetic association studies meta-analysis, covering subgroup analysis, heterogeneity test, Hardy-Weinberg analysis, analysis of publication bias, statistical relationship for the various genetic models, and robustness evaluation of the outcomes.

\section{Results}

\section{Study characteristics}

Figure 1 presents the flow charts of the selected articles. Via extensive search, 100 studies were identified. Upon elimination of duplicated articles, there were 65 articles available for title and abstract screening. Owing to inadequate information, we further omitted 40 studies which were not relevant to our study after title and abstract glancing and 8 fulltext publications which were not case-control. Since Liu et al. and Seedhouse et al. reported the findings on various subpopulations, our meta-analysis viewed each population group as a separate sample. A total
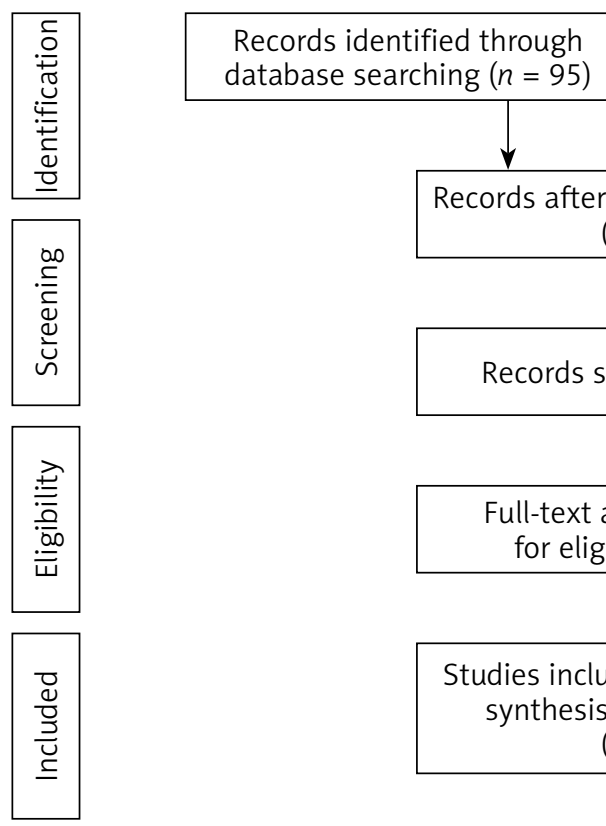

Additional records identified

through other sources $(n=5)$

Records after duplicates removed
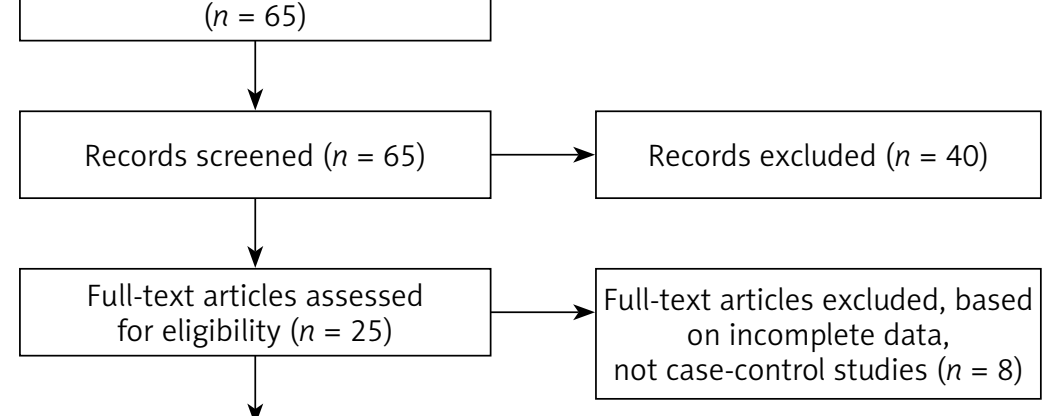

Studies included in quantitative synthesis (meta-analysis) $(n=17)$

Figure 1. Selection of the final selected studies 
of 17 studies consisting of 7241 controls and 4198 cases [36-52] met our inclusion requirements. Table I presents the characteristics of the eligible articles. Of the 17 eligible articles, twelve studies were carried out in Caucasians, four in Asians, one in persons of mixed descent and three in Africans. In addition, different studies investigated different types of leukaemia: four studies assessed acute lymphoblastic leukaemia (ALL), two investigated chronic lymphocytic leukaemia (CLL), thirteen investigated acute myeloid leukaemia and one assessed chronic myeloid leukaemia. When classified by the source of the controls, seventeen were population-based, and three were hospital-based. In all the included studies, the genotype distribution frequencies across the controls were consistent with Hardy-Weinberg equilibrium. We evaluated the link between Thr241Met polymorphism of XRCC3 and leukaemia risk susceptibility by evaluating the odds ratio (OR) and its $95 \%$ confidence interval $(\mathrm{Cl})$ among these five genetic variants: homozygote model (TT vs. CC), allele model ( $T$ vs. $C$ ), recessive model (TT vs. $T C+C C$ ), dominant model ( $T T+T C$ vs. CC), and over-dominant model (TC vs. TT+CC). No significant association was ob- served between leukaemia risk and Thr241Met polymorphism of XRCC3 across the five genetic variants ( $T$ vs. C: $\mathrm{OR}=0.871,95 \% \mathrm{Cl}: 0.606-1.251, p=0.452$; TT vs. TC+CC: $\mathrm{OR}=0.881,95 \% \mathrm{Cl}: 0.534-1.455$, $p=0.622$; TT+TC vs. CC: $\mathrm{OR}=0.842,95 \% \mathrm{Cl}: 0.629$ $1.127, p=0.247$; TC vs. TT+CC: $\mathrm{OR}=1.247,95 \% \mathrm{Cl}$ : $0.894-1.738, p=0.194 ;$ TT vs. CC: $\mathrm{OR}=0.778$, $95 \% \mathrm{Cl}: 0.492-1.230, p=0.282$ ). Figures $2-4$ represent overall pooled odds ratio in the three selected genetic models. The genotype data for Thr241Met polymorphism are presented in Table II.

\section{Quantitative synthesis}

In our ethnicity subgroup evaluation, we noted a significant protective effect of Thr241Met polymorphism of XRCC3 among Asians under four genetic models (T vs. C: $\mathrm{OR}=0.261,95 \% \mathrm{Cl}: 0.131-0.520$, $p=0.001$; TT vs. TC+CC: OR $=0.250,95 \% \mathrm{Cl}: 0.112-$ $0.558, p=0.001$; TT+TC vs. CC: $\mathrm{OR}=0.081,95 \% \mathrm{Cl}:$ $0.019-0.359, p=0.001$; TT vs. CC: $\mathrm{OR}=0.062,95 \% \mathrm{Cl}$ : $0.013-0.288, p=0.001$ ) (Table III). Nevertheless, the oncogenic potential of Thr241Met polymorphism of XRCC3 among Asians was noted under one genetic model (TC vs. TT+CC: OR $=2.053$, 95\% Cl: 0.843-

Table I. Included study characteristics

\begin{tabular}{|c|c|c|c|c|c|c|}
\hline Author & Year & Country & Ethnicity & Cancer type & Source & $\begin{array}{c}\text { Genotyping } \\
\text { method }\end{array}$ \\
\hline Fekry & 2018 & Egypt & African & ALL & PB & TaqMan \\
\hline Pei & 2018 & China & Asian & ALL & PB & PCR-RFLP \\
\hline Mutlu & 2015 & Turkey & Caucasian & CLL & PB & PCR-RFLP \\
\hline Smolkova & 2014 & Germany & Caucasian & ALL & PB & TaqMan \\
\hline Banescu & 2014 & Romania & Caucasian & CML & PB & PCR-RFLP \\
\hline Banescu & 2013 & Romania & Caucasian & AML & PB & PCR-RFLP \\
\hline Sorour & 2013 & Egypt & African & AML & $\mathrm{HB}$ & PCR-RFLP \\
\hline Abramenko & 2012 & Ukraine & Caucasian & CLL & PB & PCR-RFLP \\
\hline Yang & 2011 & China & Asian & AML & PB & PCR-RFLP \\
\hline Liu & 2011 & China & Asian & AML & PB & PCR-RFLP \\
\hline Liu & 2011 & China & Asian & $\mathrm{AML}$ & $\mathrm{HB}$ & PCR-RFLP \\
\hline Hamdy & 2011 & Egypt & African & AML & $\mathrm{HB}$ & PCR-RFLP \\
\hline Bhatla & 2008 & USA & Mixed & AML & PB & TaqMan \\
\hline Guillem & 2007 & Spain & Caucasian & $\mathrm{AML}$ & PB & PCR-RFLP \\
\hline Voso & 2007 & Italy & Caucasian & AML & PB & PCR-RFLP \\
\hline Matullo & 2006 & Mixed & Caucasian & ALL & PB & TaqMan \\
\hline Seedhouse & 2004 & UK & Caucasian & AML & PB & PCR-RFLP \\
\hline Seedhouse & 2004 & UK & Caucasian & $\mathrm{AML}$ & PB & PCR-RFLP \\
\hline Seedhouse & 2002 & UK & Caucasian & AML & PB & PCR-RFLP \\
\hline Seedhouse & 2002 & UK & Caucasian & $\mathrm{AML}$ & PB & PCR-RFLP \\
\hline
\end{tabular}




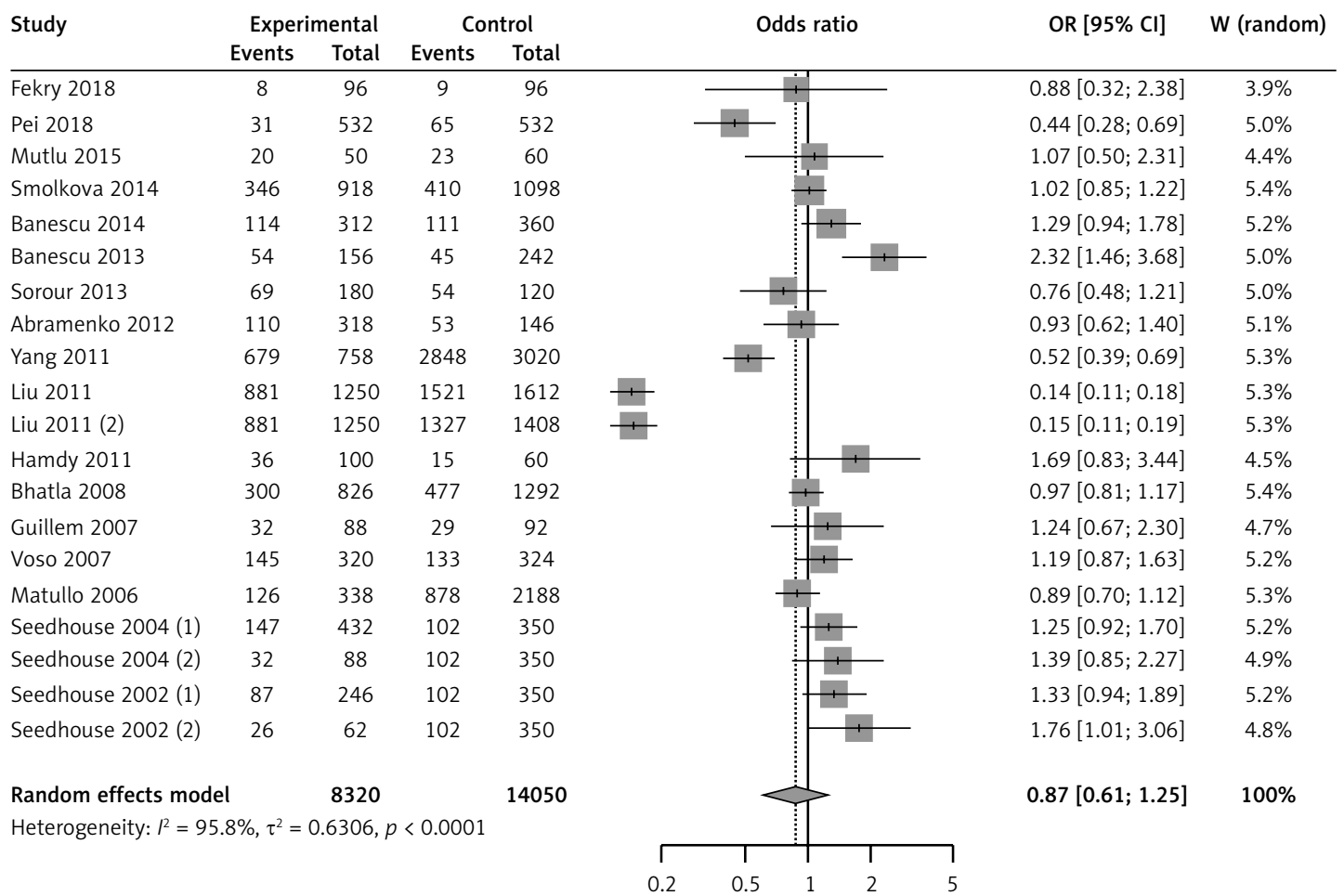

Figure 2. Overall pooled odds ratio for allele model

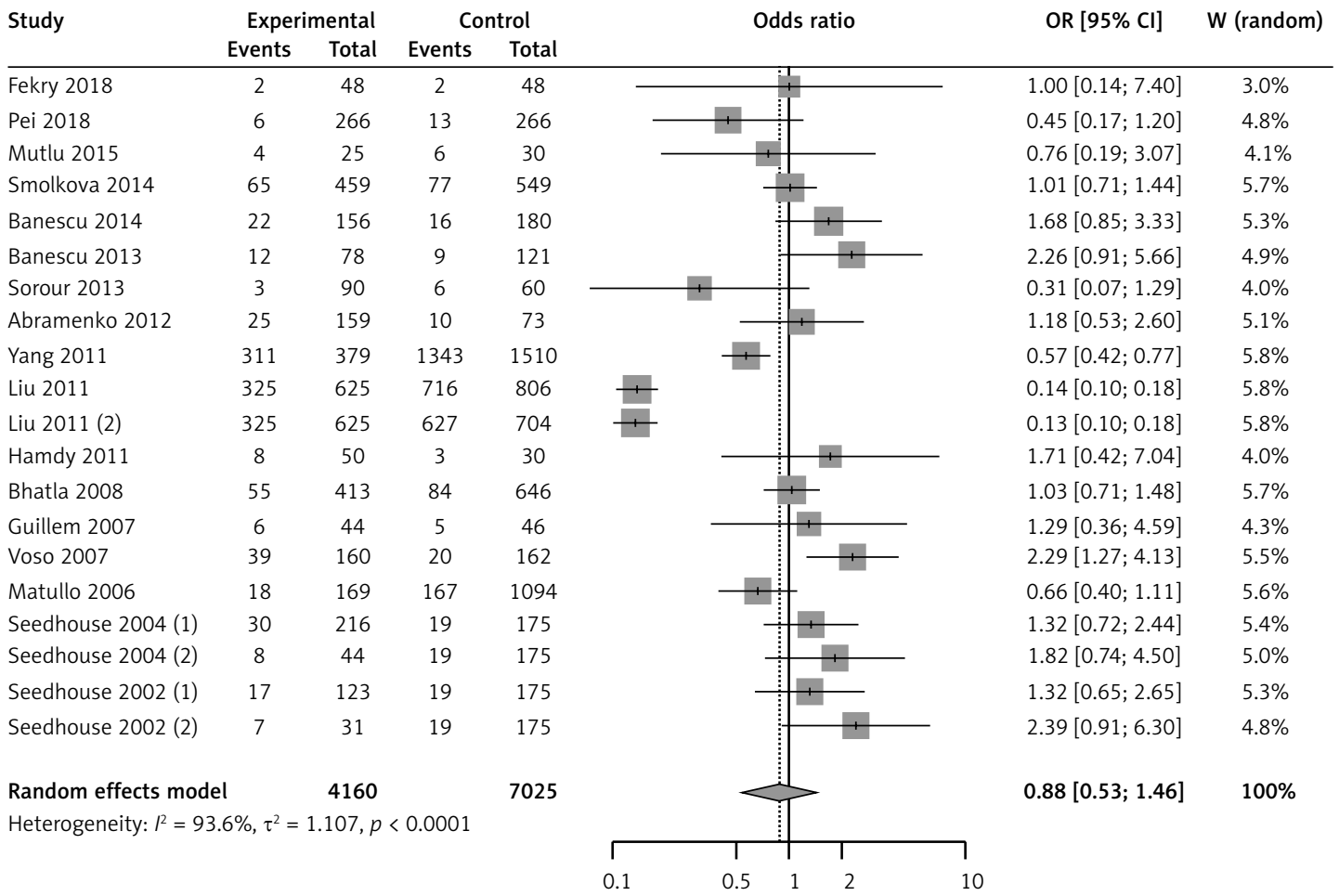

Figure 3. Overall pooled odds ratio for recessive model

4.997, $p=0.159)$. Regarding Caucasians, we observed oncogenic potential of Thr241Met polymorphism of XRCC3 under four genetic models (T vs. C: $\mathrm{OR}=1.206,95 \% \mathrm{Cl}: 1.045-1.393, p=0.010 ;$ TT vs. TC+CC: $\mathrm{OR}=1.255,95 \% \mathrm{Cl}: 1.037-1.518, p=0.020$; TT+TC vs. $\mathrm{CC}: \mathrm{OR}=1.159,95 \% \mathrm{Cl}: 1.015-1.322$, $p=0.029 ;$ TT vs. CC: $\mathrm{OR}=1.298,95 \% \mathrm{Cl}: 1.058-1.593$, $p=0.001$ ). No association was observed among Africans and persons of mixed ethnicity. No substantial association was found between XRCC3 Thr241Met polymorphism and risk of leukaemia when the source of control was taken into consideration. Our 


\begin{tabular}{|c|c|c|c|c|c|c|c|}
\hline \multirow[t]{2}{*}{ Study } & \multicolumn{2}{|c|}{ Experimental } & \multicolumn{2}{|c|}{ Control } & \multirow[t]{2}{*}{ Odds ratio } & \multirow[t]{2}{*}{ OR $[95 \% \mathrm{Cl}]$} & \multirow[t]{2}{*}{ W (random) } \\
\hline & Events & Total & Events & Total & & & \\
\hline Fekry 2018 & 2 & 44 & 2 & 43 & & $0.98[0.13 ; 7.26]$ & $2.9 \%$ \\
\hline Pei 2018 & 6 & 247 & 13 & 227 & & $0.41[0.15 ; 1.10]$ & $5.1 \%$ \\
\hline Mutlu 2015 & 4 & 13 & 6 & 19 & - & $0.96[0.21 ; 4.42]$ & $3.9 \%$ \\
\hline Smolkova 2014 & 65 & 243 & 77 & 293 & & $1.02[0.70 ; 1.51]$ & $6.4 \%$ \\
\hline Banescu 2014 & 22 & 86 & 16 & 101 & & $1.83[0.89 ; 3.76]$ & $5.8 \%$ \\
\hline Banescu 2013 & 12 & 48 & 9 & 94 & + & $3.15[1.22 ; 8.12]$ & $5.2 \%$ \\
\hline Sorour 2013 & 3 & 27 & 6 & 18 & +1 & $0.25[0.05 ; 1.18]$ & $3.8 \%$ \\
\hline Abramenko 2012 & 25 & 99 & 10 & 40 & & $1.01[0.43 ; 2.36]$ & $5.5 \%$ \\
\hline Yang 2011 & 311 & 322 & 1343 & 1348 & 1 & $0.11[0.04 ; 0.31]$ & $4.9 \%$ \\
\hline Liu 2011 & 325 & 394 & 716 & 717 & 1 & $0.01[0.00 ; 0.05]$ & $3.0 \%$ \\
\hline Liu 2011 (2) & 325 & 394 & 627 & 631 & $\longrightarrow$ & $0.03[0.01 ; 0.08]$ & $5.0 \%$ \\
\hline Hamdy 2011 & 8 & 30 & 3 & 21 & 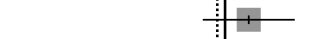 & $2.18[0.50 ; 9.45]$ & $4.0 \%$ \\
\hline Bhatla 2008 & 55 & 223 & 84 & 337 & + & $0.99[0.67 ; 1.46]$ & $6.4 \%$ \\
\hline Guillem 2007 & 6 & 24 & 5 & 27 & 1 & $1.47[0.38 ; 5.60]$ & $4.3 \%$ \\
\hline Voso 2007 & 39 & 93 & 20 & 69 & + & $1.77[0.91 ; 3.44]$ & $5.9 \%$ \\
\hline Matullo 2006 & 18 & 79 & 167 & 550 & & $0.68[0.39 ; 1.18]$ & $6.1 \%$ \\
\hline Seedhouse 2004 (1) & 30 & 129 & 19 & 111 & + & $1.47[0.77 ; 2.79]$ & $5.9 \%$ \\
\hline Seedhouse 2004 (2) & 8 & 28 & 19 & 111 & , & $1.94[0.74 ; 5.04]$ & $5.2 \%$ \\
\hline Seedhouse 2002 (1) & 17 & 70 & 19 & 111 & 1 & $1.55[0.74 ; 3.24]$ & $5.7 \%$ \\
\hline Seedhouse 2002 (2) & 7 & 19 & 19 & 111 & + & $2.82[0.98 ; 8.11]$ & $5.0 \%$ \\
\hline \multicolumn{2}{|c|}{ Random effects model } & 2612 & & 4979 & 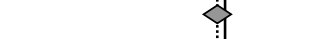 & $0.78[0.49 ; 1.23]$ & $100 \%$ \\
\hline \multicolumn{8}{|c|}{ Heterogeneity: $I^{2}=83.7 \%, \tau^{2}=0.8146, p<0.0001$} \\
\hline & & & & & 1 & & \\
\hline & & & & & 0.1 & & \\
\hline
\end{tabular}

Figure 4. Overall pooled odds ratio for homozygous model

subgroup evaluation on the various types of leukaemia also showed no association between leukaemia risk (acute lymphoblastic leukaemia, acute myeloid leukaemia, chronic myeloid leukaemia and chronic lymphocytic leukaemia) and XRCC3 Thr241Met polymorphism under the various genetic models.

\section{Publication bias and sensitivity analysis}

Begg's funnel plot and Egger's test were used to determine the publication bias of the eligible articles used in our research. The symmetrical funnel plot for the different genetic models demonstrated that the results of our evaluation have not been influenced by selection bias (Figure 5). In addition, the results of the Egger test showed that there was no substantial bias among our eligible articles, because the $p$-values for all genetic models were larger than 0.05 ( $p=0.257$ for the homozygous model; $p=0.295$ for the overdominant model; $p=0.151$ for the dominant model; $p=0.974$ for the recessive model; $p=0.365$ for the allele model). Sensitivity was then analysed to evaluate the stability of our study findings (Figure 6). When a specific study was excluded, the statistical significance of the findings was not altered, confirming the validity and reliability of our study findings.

\section{Discussion}

Numerous molecular genomic research regarding the association of Thr241Met polymorphisms of XRCC3 with leukaemia risk have been conducted in various ethnic groups, but the findings are relative- ly ambiguous. The ambiguity among studies could be due to different sources of controls, research methodology and genetic ethnicities. In addition, it is understood that single studies with small sample size might not provide sufficient statistical power to evaluate minor risk factors. Hence, meta-analyses have the merit of pooling results from several relevant studies to achieve a more accurate estimation for possible genetic associations. We therefore performed this current meta-analysis comprising seventeen studies with the objective of presenting a precise and comprehensive conclusion.

Our study is the first comprehensive update analysis on the association between leukaemia risk and Thr241Met polymorphism of XRCC3. Seventeen individual case-control studies with 7061 controls and 4198 cases were included. Overall, no significant relationship was observed regarding increased leukaemia risk and Thr241Met polymorphism of XRCC3. No association was further noted despite the adjustment of primary studies' odds ratio and the assessment of potential confounding variables. The narrow confidence interval observed in the overall pooled odds ratio indicates the high power of the quantitative analysis; therefore the findings are convincing and conclusive. Yan et al. conducted a meta-analysis of 7 case-control studies on the association between leukaemia and Thr241Met polymorphism [33]. Their analysis of the pooled data of 1634 controls and 1070 cases showed an association among Asians but not Caucasians. However, our results contradict the findings of Yan et al. in that the outcomes of 


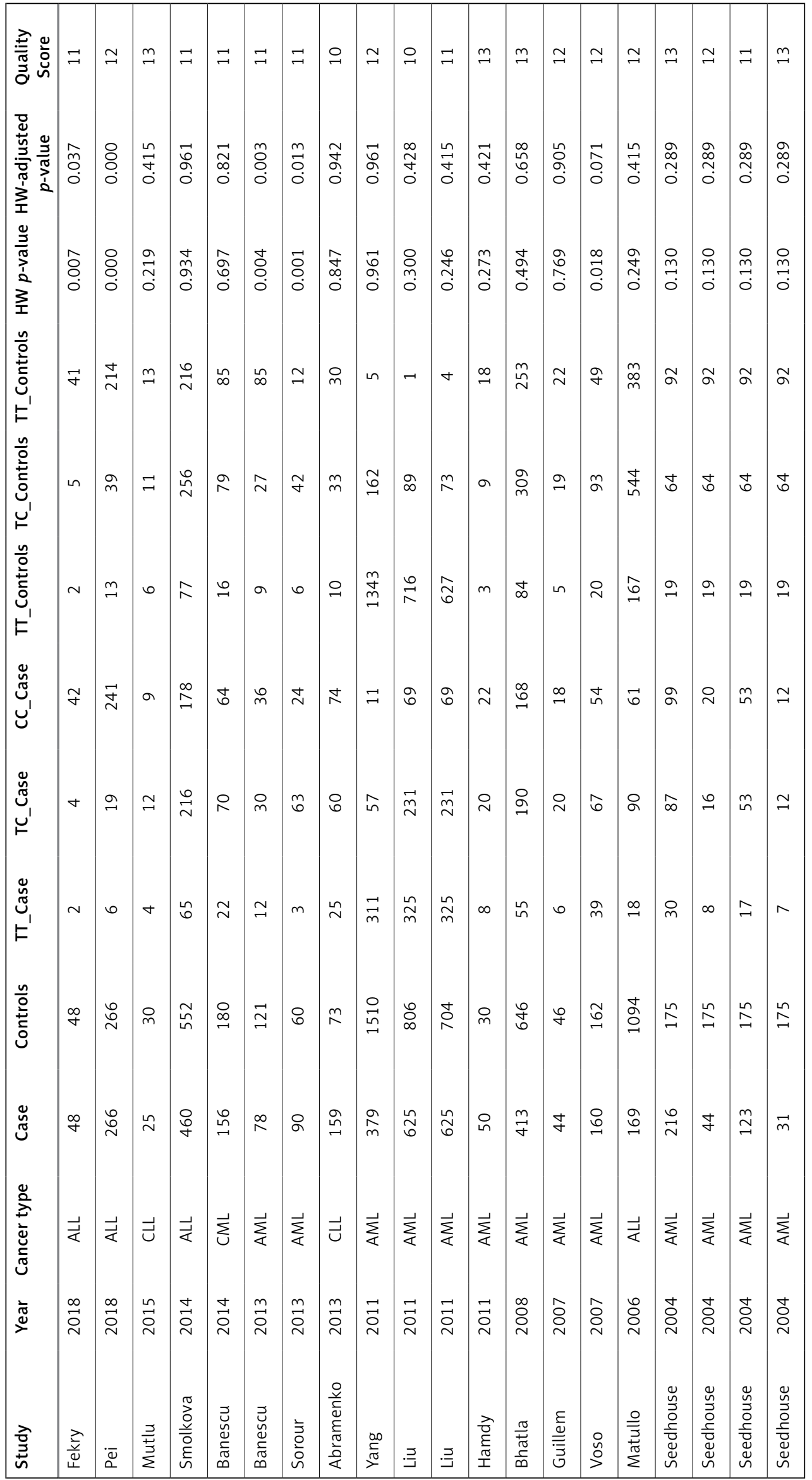




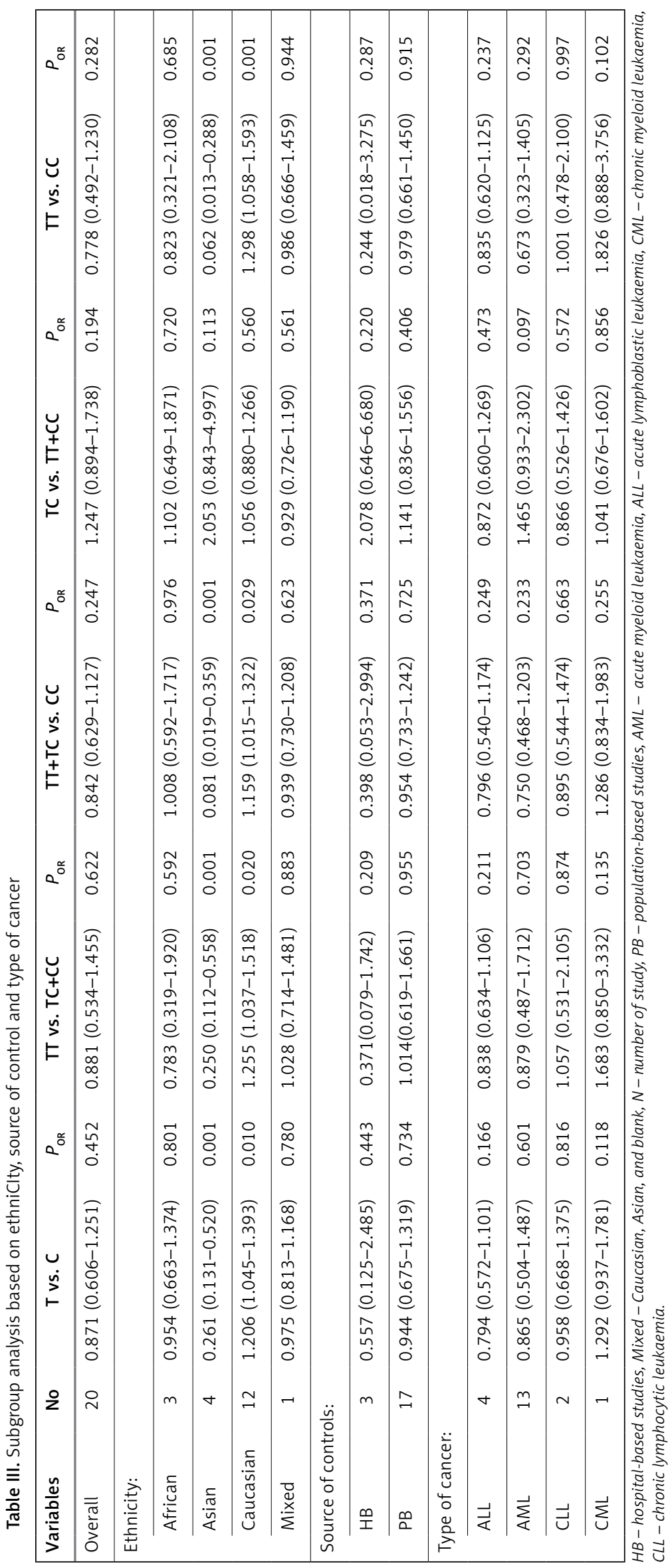


our ethnicity-based subgroup analysis revealed that Thr241Met polymorphism of XRCC3 has a protective effect in the Asian population but has oncogenic potential in Caucasians. Nevertheless, oncogenic potential of Thr241Met polymorphism of XRCC3 among Asians was noted in the over-dominant genetic model. Our findings differ from those of Yan et al. in that individual studies with limited sample size could not have sufficient statistical strength to identify or provide a fluctuated estimate of a small risk factor. Hence, even though they observed no association in Caucasians, we object to this finding on the basis of the conclusive results obtained. Again, the oncogenic potential detected in our results among Asians under the over-dominant model necessitate extra studies due to the wide confidence intervals observed.

Heterogeneity plays a significant function in meta-analyses; hence it is essential to identify the cause of any possible heterogeneity in the end results. In our meta-analysis, we found substantial heterogeneity among the various studies; hence, we applied a subgroup model to investigate the source(s) of heterogeneity. Our ethnicity-based subgroup analysis showed that heterogeneity existed among the Asian population. This can be interpreted by the hypothesis that even though they possess an identical genetic

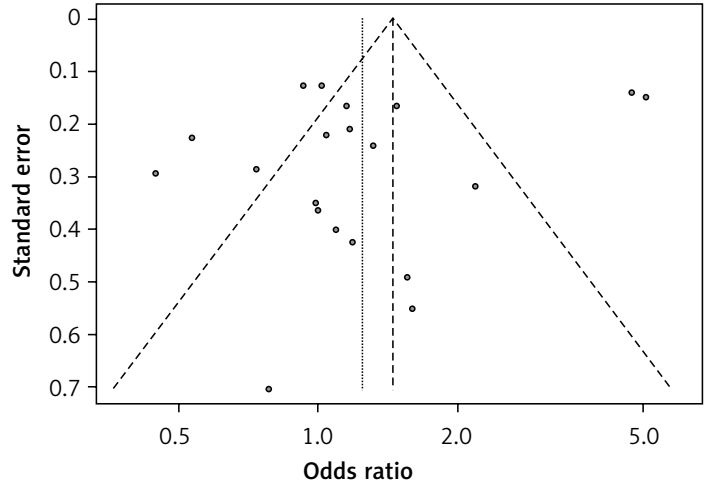

Figure 5. Funnel plot for all studies

background, different people have various habits and are prone to various risk factors, as well as different rates of risk factor exposure, which can induce heterogeneity. The stratification of the population is a disturbing problem and could contribute to snide and spurious proof on the relationship between disease and a marker, considering the differential influence of ethnicity and environmental disparities on genetic background. Our findings also confirm the findings of other studies where no association was observed between ovarian and gastric cancer risk and Thr241Met polymorphism of XRCC3 [53-55].

Study
Omitting Fekry 2018
Omitting Pei 2018
Omitting Mutlu 2015
Omitting Smolkova 2014
Omitting Banescu 2014
Omitting Banescu 2013
Omitting Sorour 2013
Omitting Abramenko 2012
Omitting Yang 2011
Omitting Liu 2011
Omitting Liu 2011 (2)
Omitting Hamdy 2011
Omitting Bhatla 2008
Omitting Guillem 2007
Omitting Voso 2007
Omitting Matullo 2006
Omitting Seedhouse 2004 (1)
Omitting Seedhouse 2004 (2)
Omitting Seedhouse 2002 (1)
Omitting Seedhouse 2002 (2)

Random effects model

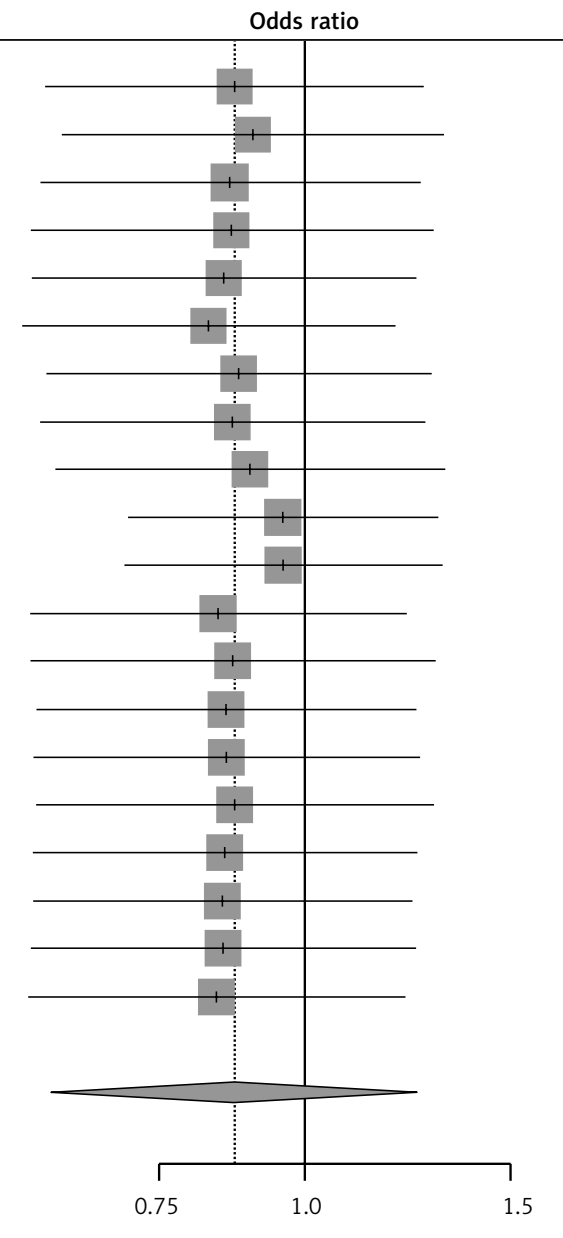

OR $95 \% \mathrm{Cl}$

$0.87[0.60 ; 1.26]$

0.90 [0.62; 1.31$]$

$0.86[0.59 ; 1.25]$

$0.86[0.58 ; 1.29]$

0.85 [0.58; 1.24]

0.83 [0.57; 1.19]

$0.88[0.60 ; 1.28]$

0.87 [0.59; 1.27]

$0.90[0.61 ; 1.32]$

$0.96[0.71 ; 1.30]$

0.96 [0.70; 1.31]

$0.84[0.58 ; 1.22]$

0.87 [0.58; 1.29]

$0.86[0.59 ; 1.24]$

$0.86[0.59 ; 1.25]$

0.87 [0.59; 1.29]

0.85 [0.58; 1.25]

0.85 [0.58; 1.24$]$

0.85 [0.58; 1.24$]$

$0.84[0.58 ; 1.22]$

$0.87[0.61 ; 1.25]$

Figure 6. Sensitivity analysis for all studies 
The DNA repair mechanism is integral to the maintenance of the stability of normal cell genomic functioning by reversing the damage to DNA. The damage of DNA at an elevated level accompanies the development and occurrence of different human diseases. XRCC3 is involved in the restoration phase of the DNA damage. Polymorphisms of XRCC3 can lead to cancer development by disrupting the repair of DNA capabilities of the protein encoded. Individuals with the genotype TT (Met/Met) could exhibit a DNA repair mechanism defect; therefore such individuals are more vulnerable to cancer, whereas individuals with one and/or two Thr alleles may have suitable mechanisms that protect against cancer.

Sensitivity analysis by repeatedly eliminating one test at a time revealed that the findings of the simplistic analysis were consistent, thus symbolizing the reliability of the study as per the findings of the sensitivity analysis. Another strength of our analysis was the consideration of adjusted odds ratio for monitoring the impact of confounding factors. Such a method confirmed our findings even further. In summary, we have conducted a high-quality quantitative analysis by following all meta-analysis steps (implementation of independent search, screening, selection, data extraction and quality score evaluation), including grey literature search and 6 databases (comprehensiveness).

Some limitations of our study exist. First, we included only Chinese and English published articles in our study, resulting in possible language bias. Secondly, subgroup analysis based on radiation exposure, gender and age was not conducted due to insufficient availability of primary studies' data. Again, in the subgroup analysis, only a handful of studies were included due to lack of information.

In conclusion, our updated quantitative evaluation of the association between leukaemia risk and XRCC3 Thr241Met polymorphism revealed no association in the overall population between Thr241Met single nucleotide polymorphism and leukaemia risk. However, we observed a protective effect in the Asian population and oncogenic potential in the Caucasian population.

\section{Conflict of interest}

The authors declare no conflict of interest.

\section{References}

1. Estey EH. Prognostic factors in acute myelogenous leukemia. Leukemia 2001; 15: 670-2.

2. Satoh Y, Matsumura I, Tanaka H, et al. C-terminal mutation of RUNX1 attenuates the DNA-damage repair response in hematopoietic stem cells. Leukemia 2012; 26: 303-11.

3. Drokow EK, Sun K, Ahmed HA, Akpabla GS, Song J, Shi M. Circulating microRNA as diagnostic biomarkers for haematological cancers: a systematic review and meta-analysis. Cancer Manag Res 2019; 11: 4313.
4. Li SY, Ye JY, Liang EY, Zhou LX, Yang M. Association between MTHFR C677T polymorphism and risk of acute lymphoblastic leukemia: a meta-analysis based on 51 case-control studies. Med Sci Monit 2015; 21: 740-8.

5. Bray F, Ferlay J, Soerjomataram I, Siegel RL, Torre LA, Jemal A. Global cancer statistics 2018: GLOBOCAN estimates of incidence and mortality worldwide for 36 cancers in 185 countries. CA J Clin 2018; 68: 394-424.

6. Chen J, Liu Y, Cai QQ, et al. Type D personality parents of children with leukemia tend to experience anxiety: the mediating effects of social support and coping style. Medicine 2015; 94: e627.

7. Arber DA, Orazi A, Hasserjian R, et al. The 2016 revision to the World Health Organization classification of myeloid neoplasms and acute leukemia. Blood 2016; 127 : 2391-405.

8. Kirtonia A, Pandya G, Sethi G, Pandey AK, Das BC, Garg $M$. A comprehensive review of genetic alterations and molecular targeted therapies for the implementation of personalized medicine in acute myeloid leukemia. J Mol Med 2020; 98: 1069-91.

9. Dong Y, Shi O, Zeng Q, et al. Leukemia incidence trends at the global, regional, and national level between 1990 and 2017. Exp Hematol Oncol 2020; 9: 14.

10. Kipps TJ, Stevenson FK, Wu CJ, et al. Chronic lymphocytic leukaemia. Nat Rev Dis Primers 2017; 3: 16096.

11. Descatha A, Jenabian A, Conso F, Ameille J. Occupational exposures and haematological malignancies: overview on human recent data. Cancer Causes Control 2005; 16: 939-53.

12. Juliusson G, Hough R. Leukemia. Progress in tumor research. 2016; 43: 87-100.

13. Short NJ, Rytting ME, Cortes JE. Acute myeloid leukaemia. Lancet 2018; 392: 593-606.

14. Thompson LH, Schild D. Recombinational D NA repair and human disease. Mutat Res 2002; 509: 49-78.

15. Szatkowska M, Krupa R. Regulation of DNA damage response and homologous recombination repair by microRNA in human cells exposed to ionizing radiation. Cancers 2020; 12: 1838.

16. Khanna KK, Jackson SP. DNA double-strand breaks: signaling, repair and the cancer connection. Nat Genet 2001; 27: 247-54.

17. Dellino GI, Palluzzi F, Chiariello AM, et al. Release of paused RNA polymerase II at specific loci favors DNA double-strand-break formation and promotes cancer translocations. Nat Genet 2019; 51: 1011-23.

18. Bishop DK, Ear U, Bhattacharyya A, et al. X rcc3 is required for assembly of Rad51 complexes in vivo. J Biol Chem 1998; 273: 21482-8.

19. Schild D, Lio YC, Collins DW, et al. Evidence for simultaneous protein interactions between human Rad51 paralogs. J Biol Chem 2000; 275: 16443-9.

20. Masson JY, Stasiak AZ, Stasiak A, et al. Complex formation by the human RAD51C and XRCC3 recombination repair proteins. Proc Natl Acad Sci USA 2001; 98: 8440-6.

21. Yamada NA, Hinz JM, Kopf VL, et al. XRCC3 ATPase activity is required for normal XRCC3-Rad51C complex dynamics and homologous recombination. J Biol Chem 2004; 279: 23250-4.

22. Xu Y, Xu D. Repair pathway choice for double-strand breaks. Essays Biochem 2020; 64: 765-77.

23. Aka P, Mateuca R, Buchet JP, et al. A re genetic polymorphisms in OGG1, XRCC1 and XRCC3 genes predictive for the DNA strand break repair phenotype and genotoxicity in workers exposed to low dose ionising radiations? Mutat Res 2004; 556: 169-81. 
24. Angelini S, Kumar R, Carbone F, et al. Micronuclei in humans induced by exposure to low level of ionizing radiation: influence of polymorphisms in DNA repair genes. Mutat Res 2005; 570: 105-17.

25. Shen M, Hung RJ, Brennan P, et al. Polymorphisms of the DNA repair genes XRCC1, XRCC3, XPD, interaction with environmental exposures, and bladder cancer risk in a case-control study in northern Italy. Cancer Epidemiol Biomarkers Prev 2003; 12: 1234-40.

26. Howlader NR, Rahman MM, Hossain MA, et al. Genetic polymorphisms in DNA repair genes XRCC1 and 3 are associated with increased risk of breast cancer in Bangladeshi population. Breast Cancer Res Treat 2020; 183: 739-50.

27. Kaur J, Sambyal V, Guleria K, et al. Association of XRCC1, XRCC2 and XRCC3 gene polymorphism with esophageal cancer risk. Clin Exp Gastroenterol 2020; 13: 73-86.

28. Mittal RD, Gangwar R, Mandal RK, Srivastava P, Ahirwar DK. Gene variants of XRCC4 and XRCC3 and their association with risk for urothelial bladder cancer. Mol Biol Rep 2012; 39: 1667-75.

29. Patil MN, Datkhile KD, Gudur A, Gudur RA, Kakade SV, Kadam SS. Polymorphism in XRCC1, XRCC2, XRCC3 genes and risk of gastrointestinal cancer: a case-control study from South-Western Maharashtra. Indian J Public Health Res Developm 2020; 11: 954-63.

30. Alkasaby MK, Abd El-Fattah Al, Ibrahim IH, Abd El-Samie HS. Polymorphism of XRCC3 in Egyptian breast cancer patients. Pharmacogenom Personalized Med 2020; 13: 273-82.

31. Qin L, Chen X, Li P, Yang Z, Mo W. Comprehensive assessment of the association between DNA repair gene XRCC3 Thr241Met polymorphism and leukemia risk. Tumor Biol 2014; 35: 2521-8.

32. Li C, Liu Y, Hu Z, Zhou Y. Genetic polymorphisms of RAD51 and XRCC3 and acute myeloid leukemia risk: a meta-analysis. Leuk Lymphoma 2014; 55: 1309-19.

33. Yan Y, Liang H, Li T, et al. Association of XRCC3 Thr241Met polymorphism and leukemia risk: evidence from a meta-analysis. Leuk Lymphoma 2014; 55: 2130-4.

34. Drokow EK, Chen Y, Waqas Ahmed HA, et al. The relationship between leukemia and TP53 gene codon Arg72Pro polymorphism: analysis in a multi-ethnic population. Future Oncol 2020; 16: 923-37.

35. Bai Y, Drokow EK, Waqas Ahmed HA, et al. The relationship between methionine synthase rs1805087 polymorphism and hematological cancers risk. Future Oncol 2020; 16: 2219-33.

36. Bănescu C, Tilinca M, Benedek EL, et al. XRCC3 Thr241Met polymorphism and risk of acute myeloid leukemia in a Romanian population. Gene 2013; 526: 478-83.

37. Sorour A, Ayad MW, Kassem $H$. The genotype distribution of the XRCC1, XRCC3, and XPD DNA repair genes and their role for the development of acute myeloblastic leukemia. Genet Test Mol Biomarkers 2013; 17: 195-201.

38. Hamdy MS, El-Haddad AM, Bahaa El-Din NM, et al. RAD51 and XRCC3 gene polymorphisms and the risk of developing acute myeloid leukemia. J Investig Med 2011; 59: 1124-30.

39. Abramenko I, Bilous N, Chumak A, et al. DNA repair polymorphisms in B-cell chronic lymphocytic leukemia in sufferers of Chernobyl Nuclear Power Plant accident. J Radiat Res 2012; 53: 497-503.

40. Liu L, Yang L, Mi Y, et al. RAD51 and XRCC3 polymorphisms: impact on the risk and treatment outcomes of de novo inv (16) or $\mathrm{t}(16 ; 16) / C B F b e t a-M Y H 11$ ( ) acute myeloid leukemia. Leuk Res 2011; 35: 1020-6.
41. Bhatla D, Gerbing RB, Alonzo TA, et al. D NA repair polymorphisms and outcome of chemotherapy for acute myelogenous leukemia: a report from the Children's Oncology Group. Leukemia 2008; 22: 265-72.

42. Seedhouse C, Faulkner R, Ashraf N, et al. Polymorphisms in genes involved in homologous recombination repair interact to increase the risk of developing acute myeloid leukemia. Clin Cancer Res 2004; 10: 2675-80.

43. Guillem VM, Collado $M$, Terol MJ, et al. Role of MTHFR $(677,1298)$ haplotype in the risk of developing secondary leukemia after treatment of breast cancer and hematological malignancies. Leukemia 2007; 21 : 1413-22.

44. Yang L, Liu L, Mi YC, et al. Relationship between RAD51G135C/ XRCC3-C241T polymorphisms and development of acute myeloid leukemia with recurrent chromosome translocation. Zhonghua Xue Ye Xue Za Zhi 2011; 32: 299-303.

45. Voso MT, Fabiani E, D'Alo F, et al. Increased risk of acute myeloid leukaemia due to polymorphisms in detoxification and DNA repair enzymes. Ann Oncol 2007; 18: 1523-8.

46. Seedhouse C, Bainton R, LewisM, et al. The genotype distribution of the XRCC1 gene indicates a role for base excision repair in the development of therapy-related acute myeloblastic leukemia. Blood 2002; 100: 3761-6.

47. Matullo G, Dunning AM, Guarrera S, et al. DNA repair polymorphisms and cancer risk in non-smokers in a cohort study. Carcinogenesis 2006; 27: 997-1007.

48. Smolkova B, Dusinska M, Hemminki K. NBN and XRCC3 genetic variants in childhood acute lymphoblastic leukaemia. Cancer Epidemiol 2014; 38: 563-8.

49. Pei JS, Chang WS, Hsu PC, et al. The contribution of XRCC3 genotypes to childhood acute lymphoblastic leukemia. Cancer Manag Res 2018; 10: 5677-84.

50. Mutlu P, Elçi MP, Yıldırım M, Nevruz O, Çetin AT, Avcu F. Identification of XRCC1 Arg399Gln and XRCC3 Thr241Met polymorphisms in a Turkish population and their association with the risk of chronic lymphocytic leukemia. Indian J Hematol Blood Transfus 2015; 31: 332-8.

51. Bănescu C, Trifa AP, Demian S, et al. Polymorphism of XRCC1, XRCC 3 , and XPD genes and risk of chronic myeloid leukemia. BioMed Res Int 2014; 2014: 213790.

52. Fekry R, Said NM, Atef W. Impact of XRCC genes polymorphisms on leukemic patients. Egypt J Pure Appl Sci 2018; 56: 1-14.

53. Yuan C, Liu X, Li R, Yan S, Kong B. Analysis of the association between the XRCC2 rs3218536 polymorphism and ovarian cancer risk. Arch Med Sci 2020; 16: 682-91.

54. Yan Y, Liang H, Li R, et al. XRCC3 Thr241Met polymorphism and ovarian cancer risk: a meta-analysis. Tumor Biology 2014; 35: 2711-5.

55. Cheng S, Wang L, Wang L, Wang Z. Association of XRCC3 gene rs861539 polymorphism with gastric cancer risk: evidence from a case-control study and a meta-analysis. Int J Clin Exp Pathol 2015; 8: 1911-9. 